that with the available evidence, particularly the financial impact, it should be more widely utilised.

\section{P208 BEHAVIOURAL FEED-BACK EDUCATION INTERVENTION TO ENHANCE ADHERENCE IN PATIENTS WITH SEVERE UNCONTROLLED ASTHMA, A RANDOMISED CLINICAL TRIAL}

'I Sulaiman, 'MC Mokoka, 'E MacHale, '1 Seheult, 'C Hughes, 'M Holmes, 'S D'arcy, ${ }^{2} \mathrm{~T}$ Taylor, ${ }^{2} \mathrm{~V}$ Rapcan, ${ }^{3} \mathrm{D}$ Murphy, ${ }^{3} \mathrm{E}$ Hunt, ${ }^{4} \mathrm{~S} J$ Lane, ${ }^{4} \mathrm{~A}$ Sahadevan, ${ }^{1} \mathrm{G}$ Crispino, ${ }^{1} \mathrm{~GB}$ Diette, ${ }^{1} \mathrm{~A}$ Sartini-Bhreathnach, ${ }^{1} \mathrm{~B}$ Cushen, ${ }^{2} \mathrm{~K}$ Killane, ${ }^{2} \mathrm{RB}$ Reilly, ${ }^{1} \mathrm{RW}$ Costello. ${ }^{1} \mathrm{RCSI}$ Beaumont Hospital, Dublin, Ireland; ${ }^{2}$ Trinity College, Dublin, Ireland; ${ }^{3}$ University College Cork, Cork, Ireland; ${ }^{4} \mathrm{AMNCH}$, Dublin, Ireland

\subsection{6/thoraxjnl-2016-209333.351}

Background Severe asthma can be due difficult to treat asthma due to poor adherence or due to refractory asthma. Identifying poor adherence can be challenging since the methods of adherence have limitations. We developed a method of assessing adherence using Inhaler compliance assessment (INCA) device, which incorporates both identifying technique errors and time of use of the inhaler. We hypothesised that that feedback on time of use and technique of use to patients, improves adherence, compared to standard education without visual feedback.

Methods This was a 3-month prospective multicentre randomised controlled stud, in which patients with severe uncontrolled asthma recruited from specialist asthma clinics were randomised to get feedback on and education using the adherence information downloaded from the INCA device or education alone.

Results At the end of the study period, the mean rate of adherence for month three in the active group $(n=111)$ was $73 \%$ versus $63 \%$ in the control group ( $\mathrm{n}=107), \mathrm{p} \leq 0.01$ (95\% CI: difference $2.8,17.6)$. Only the active group demonstrated significant reductions in the rate of technique errors missed doses, excess-doses with improvement in the habit of use. The mean AQLQ and ACT improved significantly in both groups. At the end of the study, $64(32 \%)$ patients remained poorly controlled with persistent airflow obstruction. Of these, 39 had adherence rates $<80 \%$, (mean $52 \%$ ), while 25 had adherence $>80 \%$, indicating refractory asthma.

Conclusion Without changing patients' therapy, most of the patients' asthma control improved. Two thirds of those who remained uncontrolled required further adherence counselling. $11 \%$ of the total cohort remained unstable despite adequate adherence and hence will require step up therapy. Our study shows that adherence assessment and education using INCA feedback should be considered prior to referring patients for additional therapy.

\section{P209 SPECIALIST RESPIRATORY PHARMACIST CASE MANAGEMENT COPD MEDICINES OPTIMISATION CLINICS: IMPLEMENTATION AND OUTCOMES}

${ }^{1} \mathrm{C}$ Jones, ${ }^{1} \mathrm{R}$ Miller, ${ }^{1} \mathrm{R}$ Sharkey, ${ }^{1} \mathrm{~A}$ Friel, ${ }^{1} \mathrm{D}$ Clifford, ${ }^{1} \mathrm{C}$ Darcy, ${ }^{1} \mathrm{~B}$ Moore, ${ }^{2} \mathrm{M}$ Hall. ${ }^{1}$ Western Health and Social Care Trust, Altnagelvin Area Hospital, Londonderry, N Ireland; ${ }^{2}$ School of Pharmacy, Queen's University, Belfast, N Ireland

\subsection{6/thoraxjnl-2016-209333.352}

Introduction and objectives In line with 'Transforming Your Care' (restructuring of healthcare provision in Northern Ireland) and the Global Initiative for Chronic Obstructive Lung Disease (GOLD) strategy, this project brought specialist trust pharmacist- led medicines optimisation case management clinics to COPD patients in primary care. The aim of the project was to achieve sustained medicines optimisation with associated improved patient outcomes.

Method An initial process mapping event resulted in establishing the existing COPD patient pathway between primary and secondary care. This informed the decision to base clinics in GP surgeries where the pharmacist: determined disease stage (GOLD classification); assessed medication adherence; established COPD medication appropriateness; prescribed COPD medications and smoking cessation; determined whether antibiotic prescribing was guideline-informed; and made appropriate referrals to primary and secondary care healthcare professionals. A 30-day telephone follow-up by the pharmacist involved reassessment of adherence, symptom scores and medication appropriateness. COPD exacerbations, antibiotic prescribing and unplanned hospital admissions were further recorded over 12 months. All data were analysed using SPSS Version 22.

Results Results for a patient cohort seen over four months $(\mathrm{n}=360)$ demonstrated: statistically significant improvements in COPD medication appropriateness and adherence (Wilcoxon Signed Rank Test, $\mathrm{p}<0.001, \mathrm{n}=360$ ); improvement in COPD symptoms (MRC Breathlessness and CAT score); and reduced guideline-informed antibiotic prescribing (12 months post baseline review). Projected annual drug cost savings were $£ 235 \mathrm{k}$. Sixty-eight percent of patients had experienced one or more COPD exacerbations over the year prior to clinic attendance reducing to $50 \%$ during the 12 months post-intervention. Nonelective COPD-related hospital admissions also decreased (9.2\% versus $5.3 \%$ over 12 months).

Conclusion Providing specialist hospital pharmacist COPD clinics in primary care resulted in safe and cost-effective medication use with improved patient outcomes 12 months post review.

\section{P210 IMPLEMENTATION OF ELECTRONIC COPD DISCHARGE CARE BUNDLES: A QUALITY IMPROVEMENT PROJECT}

S Santharam, ST Lugg, G Packer, R Colclough, S Gompertz. Queen Elizabeth Hospital Birmingham, Birmingham, UK

\subsection{6/thoraxjnl-2016-209333.353}

Background and aims Acute exacerbations of chronic obstructive pulmonary disease (AECOPD) are responsible for 115,000 emergency admissions to hospital p.a.; one-third of patients are readmitted within 90 days. COPD discharge care bundles reduce re-admission rate. ${ }^{1}$

Paper-based COPD discharge bundles were implemented in our trust (a large tertiary centre in the West Midlands) in 2013, with disappointing completion rates. In order to improve compliance, an electronic bundle (e-bundle) was introduced This uses a novel system (Figure 1) that electronically alerts our multidisciplinary respiratory support team to potential AECOPD patients when medications for AECOPD are electronically prescribed (eprescribed). The aim of this audit was to see whether completion of COPD discharge bundles in this trust has improved since the e-prescribing alert system and e-bundle implementation in 2015. Methods Admissions coded as AECOPD were retrospectively identified using ICD-10 codes for a three-month continuous period in 2015. Discharge summaries and e-bundles for each patient were accessed via online records, and in patients who were bundle-appropriate, data were collected on the presence or 